\title{
Iodine-Assisted Laser Desorption/Ionization Time-of-Flight Mass Spectrometry
}

\author{
Sajid Bashirr,2, Sai Raghuveer Chava ${ }^{3,4, *}$, Jingbo L Liü ${ }^{2,4}$, and Peter J Derrick ${ }^{1,5}$,\# \\ ${ }^{1}$ Institute of Mass Spectrometry, University of Warwick, UK \\ ${ }^{2}$ Department of Chemistry, Texas A\&M University, USA \\ ${ }^{3}$ Emergent BioSolutions, USA \\ ${ }^{4}$ Frederick E Giesecke Engineering Research Building, Texas A\&M Energy Institute, USA \\ ${ }^{5}$ Department of Physics, The University of Auckland, USA \\ [\#] Dedicates to the memory of Peter J Derrick and Mohammed Bashir
}

Research Article

Volume 4 Issue 1

Received Date: December 20, 2020

Published Date: January 26, 2021

DOI: $10.23880 /$ aabsc-16000156

*Corresponding author: Sai Raghuveer Chava, Department of Chemistry, Texas A\&M University, 700 University Blvd., Kingsville, TX, 78414, USA, Tel: 01 8588868214; Email: ChavaS@ebsi.com; br9@tamuk.edu

\section{Abstract}

Adsorption and desorption of iodine-containing $\alpha$-cyano-4-hydroxycinnamic acid (aCCa) matrix species were studied using matrix-assisted laser desorption/ionization time-of-flight mass spectrometry (MALDI-TOF MS). The MALDI-TOF MS method showed that ca. 0.8- 1.4 monolayer $(\sim 100 \mathrm{ppm})$ of iodine-containing species was adsorbed at the surface and assisted in desorption and ionization of a protein digest peptides or peptides varying in isoelectric potential. At low laser power, desorption of analytes in protonated and sodiated form was observed but not iodine cluster relative to aCCa without iodine, suggesting a two-electron reduction process to form the protonated pseudo molecular ion, although adsorption on the surface would lead to oxidation of iodide to iodine. The addition of iodine to matrix has been demonstrated to greatly facilitate the MALDI-TOF MS process and is a valuable tool when complex protein mixtures need to be analyzed.

Keywords: MALDI; $\alpha$-cyano-4-hydroxycinnamic acid; Desorption; Ionization; Mass Spectrum

\section{Introduction}

Ultraviolet Matrix-assisted laser-desorption/ionization (UV MALDI summarized in Figure 1) has become an important analytical technique in the characterization of a range of compounds. Under MALDI conditions, a UV absorbing molecule (the 'matrix') is pre-mixed with the analyte under investigation. The binary mixture is deposited onto a stainless-steel slide and allowed to dry. Once dry, a UV laser $(\lambda=337 \mathrm{~nm})$ is fired, and ions are generated. On average, the penetration depth of a typical nitrogen laser is estimated to be about $85 \mathrm{~nm}$, thus the excitation profile is essentially two-dimensional [1]. Many problems remain with MALDI. These can be grouped into problems with (a) poor desorption and (b) poor ionization collectively leading to low signal intensity for the analyte.

\section{Desorption Problems}

Two pressing problems are lack of 'universal matrix' compounds that can be co-mixed with any type of analyte species and large variation in the analyte signal intensity. The lack of a suitable matrix for any analyte is due to the unfamiliarity of what constitutes a 'good' matrix. Certain physical parameters such as proton affinity (PA), gas-phase basicity (GB), sublimation enthalpy, and crystal structure or absorption coefficient are thought to be important but have not been examined in a systematic manner [2]. In the report, evaluation of iodine against alpha cyano cinnamic acid as the ultraviolet-absorbing matrix since it can be used in the analysis of peptides, protein, and carbohydrates (Figure 1). The analytes evaluated were model neuropeptide, cyclic oligosaccharide, oxygen-rich polymer, and a small molecule. 
The problem with analyte ion variation is due to the randomness in analyte-matrix mixing and deposition. For an efficient analyte signal, an optimal ratio of matrix-to-analyte molecules is required. This optimal ratio depends on analyte size, structure, and $\mathrm{pH}$. Due to the statistical nature of mixing, some regions have excess matrix or analyte or no matrix (known as 'bald spots'). Therefore, during irradiation, where the matrix-and-analyte is deposited in the correct ratio, the analyte signal is observed and, where there is an insufficient matrix, no signal is observed. The sum of these signals can give rise to large variances. Previously, we attempted using a copper surface with laser-etched 'pits' as an ordered array which was promising in ordering the matrix but complicated in the etching of the array (Figure 2). Our approach is to apply a polyphenylene-based dendrimer as an anchor on which our matrix is 'ordered.' We believe this ordering (between the surface and matrix) will be through Van der Waal or charge-charge interactions and will accomplish the desired matrix ordering. Recently, carbon nanotubes have been used in the desorption of proteins, which is similar in that it allows a sorptive anchor for the protein [3].

\section{Ionization Problems}

Once the matrix has been desorbed from the surface the matrix and analyte molecules interact in the gas phase leading to ionization of the analyte; the precise manner in which this transfer occurs and in what (geometric/energy) state the matrix species is not known. Although speculative, it is hypothesized that enhancement of the analyte peak intensity can occur through efficient protonation, presumably due to lower ion neutralization from the excess electrons generated at the surface [4]. The probable routes for energy transfer from the matrix to analyte are: (i) exciton pooling of the matrix molecule and (ii) generation of negative matrix ions through electron emission from the surface $(\sim 10 \mathrm{~nm})$ and possible charging effect of electron emission at the surface/ vacuum interface. Analysis of the proton affinity (PA) and gas-phase basicity (GB) of alpha cyano cinnamic acid suggest that ground-state proton transfer from the matrix radical cations to the analyte may be important in efficient analyte ionization. Therefore, one might speculate that electron emission from the surface, followed by electron capture may contribute to the generation of matrix radical cations that, independently or in tandem with matrix exciton pooling, would give rise to efficient analyte ionization and signal. Since measurement of the gas-phase proton affinities of matrices is a difficult procedure, computational methods were investigated to determine whether high-level density functional theory (DFT) could be applied. We previously used the experimental PA/GB values for $\mathrm{x}, \mathrm{y}$-DHBs generated a DFT model that could compute PA/GB values that were in close agreement with the experiment [5]. A further advantage of using a DFT approach is it can predict the PA/GB values for molecules that have not been currently synthesized. Also, the electron or sodium affinity, matrix proton linked dimer can be computed which are physically difficult to measure. From the DFT model, structures that have a propensity for form stable dimers (to promote excitation pooling) or ground state radicals can be synthesized and tested. Our second aim was to modify our existing DFT model (developed for $\mathrm{x}, \mathrm{y}$-DHBs) and apply it to the alpha cyano cinnamic acid structure. For the most efficient matrix design, we believe our absorbed matrix deposited onto the crushed matrix or placed on the copper grid may be ordered on the surface but also allow interaction with the analyte molecules for efficient analyte ionization, which would be similar to the iodine-assisted desorption platform [6-8]. The coating of the anchor and metal likewise can be followed through optical, scanning electron (SEM), and transmission electron (TEM) microscopy, respectively.

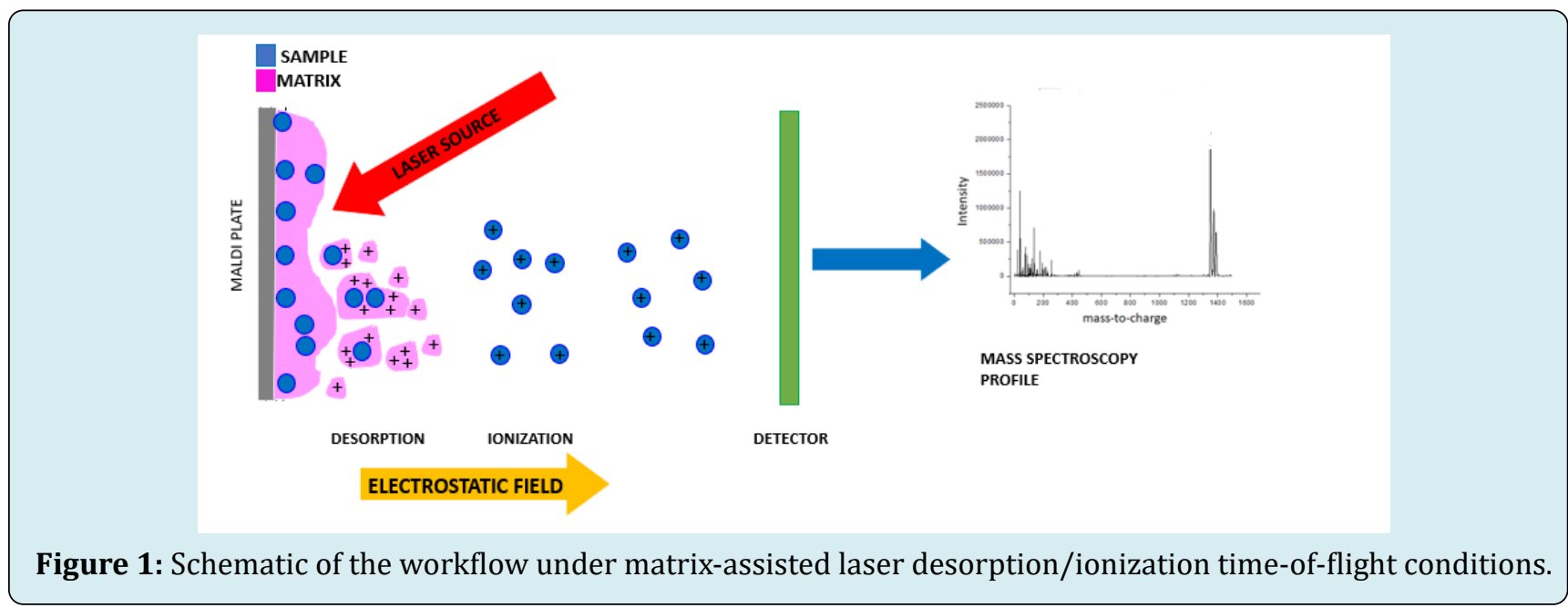

Sai Raghuveer Chava, et al. Iodine-Assisted Laser Desorption/Ionization Time-of-Flight Mass Spectrometry. Ann Adv Biomed Sci 2021, 4 (1): 000156. 


\section{Goal}

To increase analyte signal intensity and thereby increase technique sensitivity.

Methodology: All chemicals were obtained from commercial sources and used without further modification. In-house ultrapure water was used, and the solvents were classified as an analytical grade. The matrix was crystallized, crushed into a fine powder, and deposited onto the stainless-steel slide. Once dried, another aliquot of the matrix was applied to contain the analyte in a 1:1000 ratio or the ratio was adjusted to obtain the highest signal-to-noise ratio.

Density Functional Theory: The proton affinity $(\mathrm{M}+$ $\mathrm{H}+\mathrm{CMH}^{+}$) of a reaction can be defined as the negative of the enthalpy of reaction.

\{Equation $\mathrm{I}\} \mathrm{PA}=-\Delta \mathrm{H}=-(\Delta \mathrm{E}+\mathrm{RT})$;

$\{$ Equation II $\} \Delta \mathrm{E}=[\mathrm{ET}(\mathrm{MH}+)-\mathrm{ET}(\mathrm{M})]+[\mathrm{ZPE}(\mathrm{MH}+)-$

$\mathrm{ZPE}(\mathrm{M})]+3 / 2 \mathrm{RT}$ and

$\{$ Equation III $\} \cdot \mathrm{PA}=-(\Delta \mathrm{ET}+\Delta \mathrm{ZPE}+5 / 2 \mathrm{RT})$.

Using the above scheme (Equations I-III), the theoretical values of the zero-point vibrational energy, vibrational energy, and the vibrational entropy obtained at the B3LYP/6-31G* level can be modeled and scaled accordingly [9], concerning the matrix. The initial computational goal is to identify the geometrical structure for each molecular species, which corresponds to a global minimum on the potential energy surface. This is important in that if the structure gives rise to low analyte ion intensity that may be due to poor PA or geometry in the gas-phase leading to poor analyte ionization.
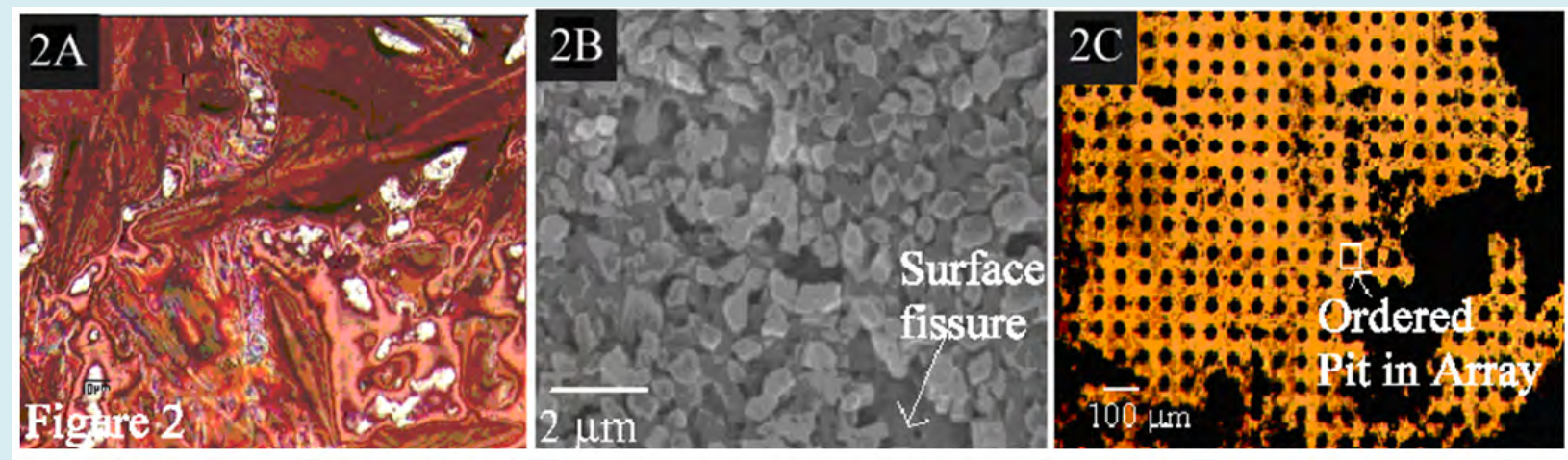

Figure 2 (2A, 2B and 2C): Strategies to eliminate ion signal variation as a function of matrix deposition on the MALDI slide.

In Figure 2A, a red dye was co-mixed with 2, 5-DHB. Regions of poor mixing (white areas), DHB crystals, and dye can be observed. By crushing the crystals and depositing them, some cracks or fissures can be observed (Figure 2B) which can be eliminated by using mechanical pits (Figure 2C). This shows matrix deposited onto a copper surface, with the pits being etched away after matrix formation, leaving the matrix deposited in an ordered array. Our current approach will be ordered the matrices using one-dimensional nanotubes, which has the advantages of ease of use and costeffectiveness compared to ordered pit array.

\section{Mass Spectrometry}

All MS measurements were carried out with a Kratos III (Coventry, U.K), with a $15-20 \mathrm{kV}$ extraction with 8-10 kV post-acceleration, using a nitrogen laser at $337 \mathrm{~nm}$. Positive mode spectra in the linear mode were sampled with a sum of ten shot spectra summed to two hundred unless otherwise noted. The laser $(\lambda=337 \mathrm{~nm})$ power was adjusted to obtain a signal-to-noise $(\mathrm{S} / \mathrm{N})$ ratio of at least three and the lowest power was used between different $\mathrm{S} / \mathrm{N}$ ratios that were three or greater. A spectrum consisting of two hundred shot was saved, using a calibration of protein digest or wide mass band (WB) peptide mixture depending on the expected mass-tocharge ratio of the sample. An extraction delay time (50-150 ns) was used in some cases to improve resolution and avoid detect saturation and 5-500 shots per spectra were used.

\section{Results}

The matrix alpha cyano-cinnamic acid (aCCa) to substance $\mathrm{P}$ without and without 100 parts-per-million mass percent iodine (dissolved in methanol) was shown under identical conditions, except laser power (LP) was adjusted to yield at least three times signal-to-noise ratio, to determine the limit of detection (LOD) is summarised in Figure 3. Each sample was deposited ten times and the signal standard deviation is also shown for each test-system. In all but two cases the signal intensity was superior for the system supplemented with iodine, and where the non-iodine system yield greater peptide intensities, the variation in the 
peptide signal was greater across the ten samples, although the average was greater. It appears that in some instances the 'sweet spot', an area of optimal matrix-to-analyte (around 1 or $10 \mathrm{nM}$ analyte) were achieved, although with iodine more uniform results were obtained.

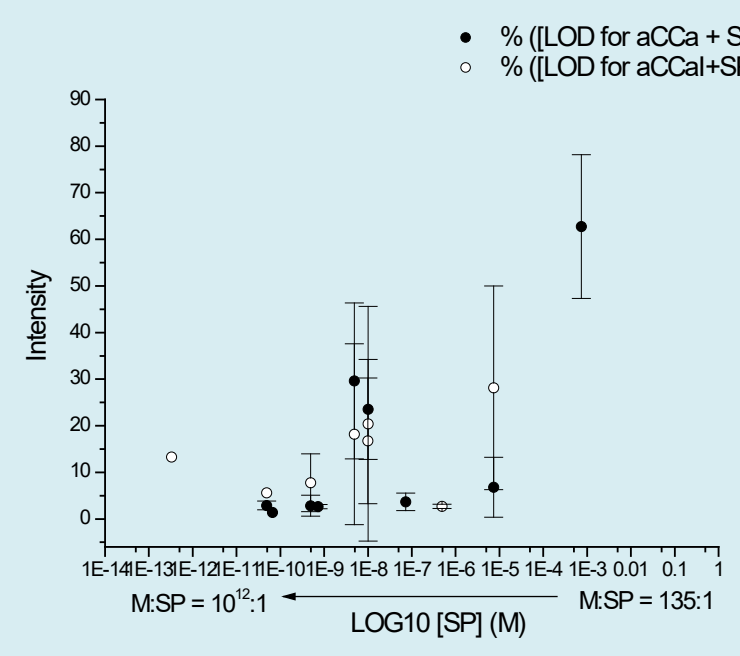

Figure 3: Iodine assisted laser desorption/ionization time-of-flight mass spectrometry corresponding to Figure 2B and matrix-assisted laser/desorption time-of-flight mass spectrometry are compared and contrasted with substance $\mathrm{P}$ was the analyte system.

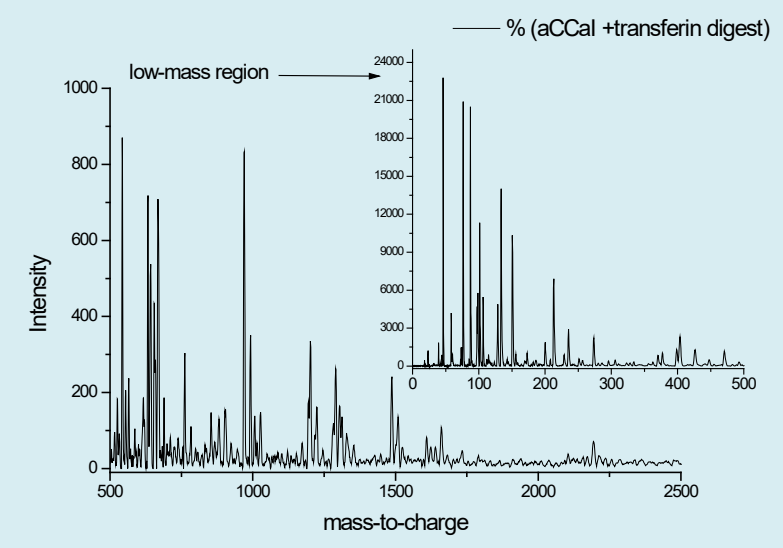

Figure 4A: Iodine assisted laser desorption/ionization time-of-flight mass spectrometry of transferrin digest. The insert is the low matrix mass region.

Human transferrin, a 698 amino acid iron carrier protein with a theoretical pI of 6.81, an average mass of 77063.89 and monoisotopic mass of 77013.63 Da was digested using trypsin at 37 oC with ammonium bicarbonate buffer and the hypothetical digests at 1500 Da summarised in Table 1 was likewise analyzed using iodine (Figure 4A) and standard aCCa matrix only (Figure 4B). All of the fragments listed in Table 1 were identified using aCCaI system but not with aCCa alone. In the aCCa, approximately $73 \%$ of the predicted peaks were observed. To system was deposited onto a copper grid (G) and the tryptic mixture was deposited, onto the grid. The grid was washed with alkali salts of sodium, potassium, and acetic acid before the addition of the digest and substance $\mathrm{P}$ (in trifluoroacetic acid, $0.01 \%$ ) was added as an external calibration standard, the results are shown in Figure 4C.

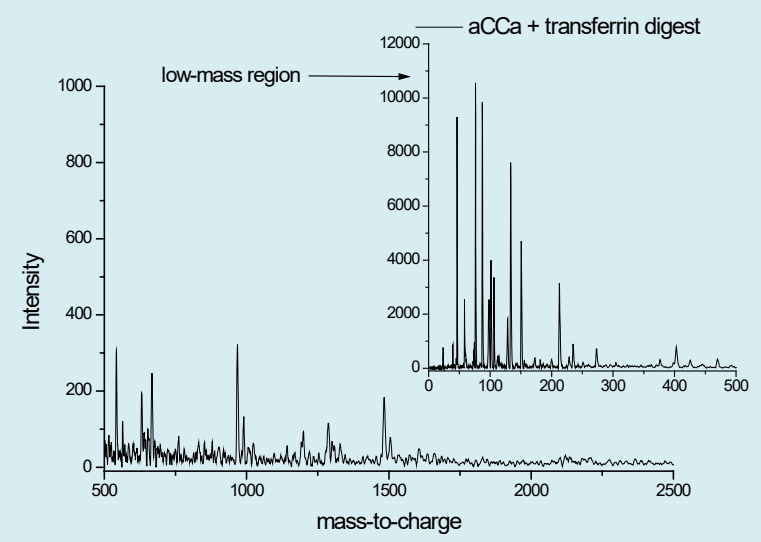

Figure 4B: Matrix-assisted laser desorption/ionization time-of-flight mass spectrometry of transferrin digest. The insert is the low matrix mass region.

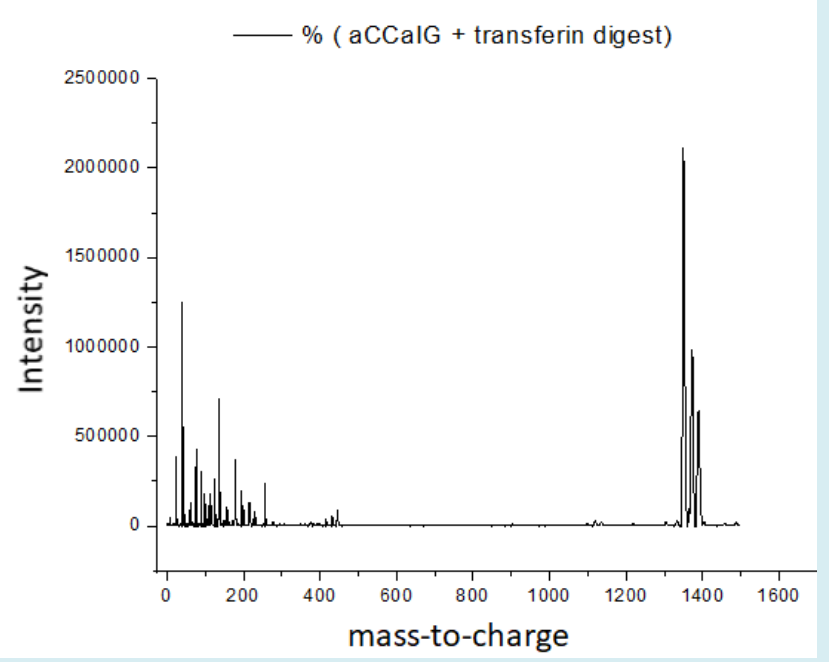

Figure 4C: Iodine-assisted laser desorption /ionization time-of-flight mass spectrometry of transferrin deposited into a metal copper grid (G) corresponding to Figure 2C and substance $\mathrm{P}$ as external calibrant. 


\begin{tabular}{|c|c|c|}
\hline Mass & Position & Peptide sequence \\
\hline 1520.636 & $495-508$ & FDEFFSEGCAPGSK \\
\hline 1482.689 & $240-251$ & DQYELLCLDNTR \\
\hline 1478.735 & $332-343$ & MYLGYEYVTAIR \\
\hline 1419.73 & $421-433$ & CGLVPVLAENYNK \\
\hline 1417.645 & $684-696$ & CSTSSLLEACTFR \\
\hline 1358.698 & $47-60$ & SVIPSDGPSVACVK \\
\hline 1297.609 & $577-587$ & DYELLCLDGTR \\
\hline 1283.569 & $531-541$ & EGYYGYTGAFR \\
\hline 1276.632 & $300-310$ & EFQLFSSPHGK \\
\hline 1273.654 & $226-236$ & HSTIFENLANK \\
\hline 1260.568 & $27-37$ & WCAVSEHEATK \\
\hline 1249.606 & $454-464$ & SASDLTWDNLK \\
\hline 1223.536 & $374-384$ & CDEWSVNSVGK \\
\hline 1195.552 & $123-132$ & DSGFQMNQLR \\
\hline 1166.591 & $554-564$ & HQTVPQNTGGK \\
\hline 1138.521 & $363-371$ & WCALSHHER \\
\hline 1000.499 & $669-676$ & YLGEEYVK \\
\hline 978.489 & $216-225$ & DGAGDVAFVK \\
\hline 964.5323 & 601-609 & APNHAVVTR \\
\hline 940.4557 & $62-69$ & ASYLDCIR \\
\hline 878.4618 & $252-258$ & KPVDEYK \\
\hline 874.4417 & $316-323$ & DSAHGFLK \\
\hline 864.4131 & $652-659$ & DDTVCLAK \\
\hline 830.3937 & $136-143$ & SCHTGLGR \\
\hline 830.3937 & $468-475$ & SCHTAVGR \\
\hline 827.4046 & $565-571$ & NPDPWAK \\
\hline 735.4035 & $547-553$ & GDVAFVK \\
\hline 686.329 & 613-618 & EACVHK \\
\hline 663.3824 & $647-651$ & DLLFR \\
\hline 654.3093 & $664-668$ & NTYEK \\
\hline 652.297 & $510-515$ & DSSLCK \\
\hline 642.314 & $490-494$ & INHCR \\
\hline 640.2871 & $38-42$ & CQSFR \\
\hline 635.3763 & $311-315$ & DLLFK \\
\hline 629.3729 & $677-682$ & AVGNLR \\
\hline 617.3253 & $572-576$ & NLNEK \\
\hline 614.3872 & $163-167$ & KPLEK \\
\hline 591.317 & $542-546$ & CLVEK \\
\hline 540.2888 & $660-663$ & LHDR \\
\hline 530.2391 & $43-46$ & DHMK \\
\hline
\end{tabular}

the protonated and alkali species for substance $\mathrm{P}$ much greater than when used without a grid system. No copper peaks were observed, and only $11 \%$ of the expected protein digest peaks were observed.

The proton affinity of alpha cyano cinnamic acid was calculated to $180 \mathrm{kcal} / \mathrm{mol}$ with an error of $3 \mathrm{kcal} / \mathrm{mol}$ and that of the amino acid glycine was $210 \mathrm{kcal} / \mathrm{mol}$ with an error of $3 \mathrm{kcal} / \mathrm{mol}$. This suggests that proton transfer from the ground state of the matrix to the analyte can occur where the proton affinity of the acceptor is greater than the donor. Other peptides are likely to have proton affinities greater than $210 \mathrm{kcal} / \mathrm{mol}$, suggesting that the excited state matrix species may be involved in addition to possible alkali metal attachment. Therefore, the parameter that may define ion yield is not the PA value but relative species that are capable of charge transfer and this tendency pay depend upon the analyte affinities, which may be related to isoelectric point (pI) values. To test this hypothesis, a less acidic protein was analyzed, sperm whale myoglobin, theoretical pI: 8.70, average mass 17331.09 , and monoisotopic mass of 17320.25 was evaluated and shown in Figure 4D and Table 2. All except one fragment sequence was observed using aCCaI and nine sequences were obtained from aCCa alone (data not shown), suggesting that assisted iodine desorption is efficient with both acidic and basic protein fragments. To investigate further, an eleven amino acid wide mass band (WB) peptide mixture was analyzed using aCCa alone and all peaks were observed. The peak intensity for the protonated species was summed and normalized to $100 \%$. The WB mix spectra are shown in Figure 4E. The same peptides were also detected using aCCaI, however, the summed peak intensities were $373 \%$ versus $100 \%$ suggesting that under near-identical conditions, greater peak intensities could be obtained using iodine supplementation.

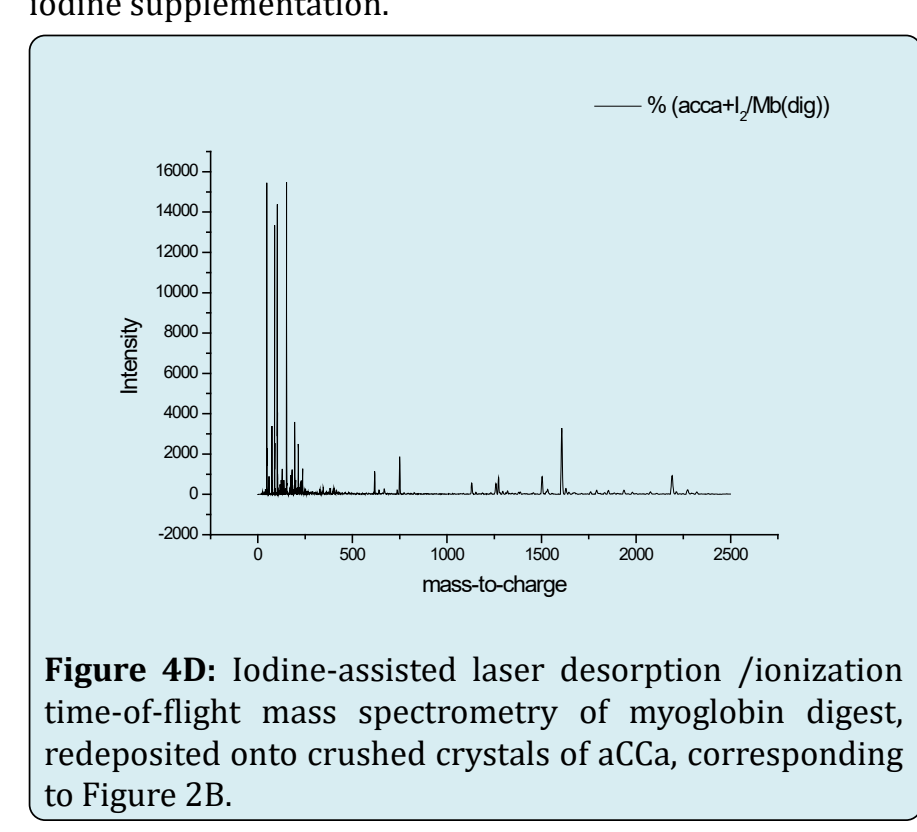

Table 1: Calculated Mass fragment, amino acid position, and predicted amino acid sequence for transferrin digested with trypsin.

One-hundred-fold increase in peak intensity was observed which dwarfed the protein digest mixture showing 


\begin{tabular}{|c|c|c|}
\hline Mass & Position & Peptide sequence \\
\hline 2025.063 & $1-17$ & MVLSEGEWQLVLHVWAK \\
\hline 1927.044 & $104-119$ & YLEFISEAIIHVLHSR \\
\hline 1853.962 & $81-97$ & GHHEAELKPLAQSHATK \\
\hline 1592.839 & $18-32$ & VEADVAGHGQDILIR \\
\hline 1515.665 & $120-134$ & HPGDFGADAQGAMNK \\
\hline 1392.857 & $65-78$ & HGVTVLTALGAILK \\
\hline 940.4734 & $36-43$ & SHPETLEK \\
\hline 748.4352 & $135-140$ & ALELFR \\
\hline 708.3232 & $52-57$ & TEAEMK \\
\hline 666.3093 & $149-154$ & ELGYQG \\
\hline 662.3355 & $58-63$ & ASEDLK \\
\hline 517.298 & $142-146$ & DIAAK \\
\hline & & \\
\hline
\end{tabular}

Table 2: Calculated Mass fragment, amino acid position, and predicted amino acid sequence for myoglobin digested with trypsin.

The wideband peptide mixture consists of peptides that have a wide variation in theoretical isoelectric points, example includes the peptide bradykinin (10.3), bombesin (7.4), dermorphin (5.7), ELEDOS (where 0=Pyrrolysine, 3.9), GGA (5.8), leucine-enkephalin (5.7), methionine-enkephalin (5.6), renin substrate (7.5), somatostatin 28 (10.3), substance $\mathrm{P}$ (11.4), and vasopressin (5.8) that span a range of $\mathrm{pI}$ from 3.9 (ELEDOS) to 11.4 (substance P).

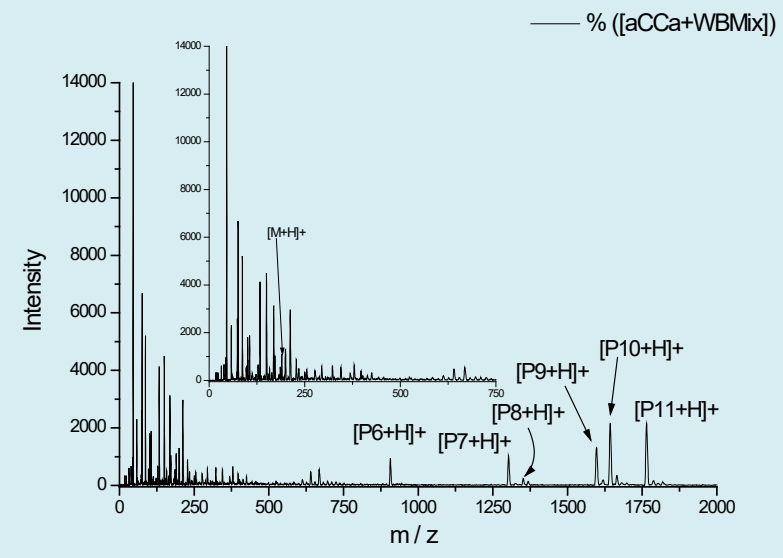

Figure 4E: Matrix-assisted laser desorption /ionization time-of-flight mass spectrometry of wide mass band (WB) peptide mixture deposited into crushed aCCa crystals corresponding to Figure 2B and summed to $100 \%$ peak intensities for the protonated species.

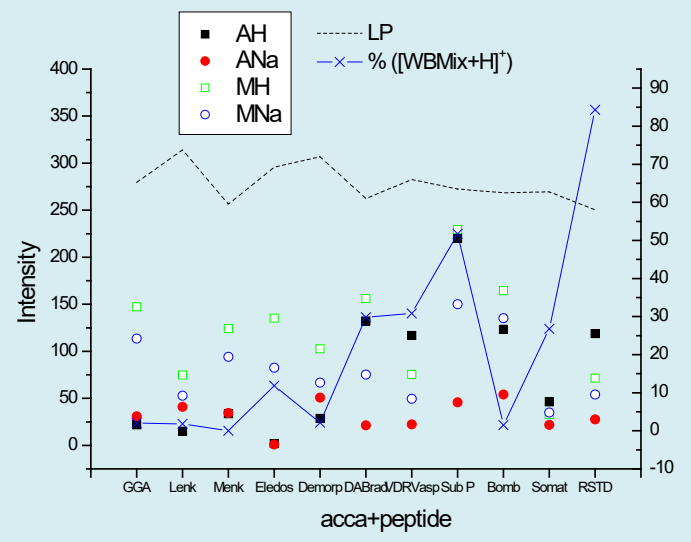

Figure 4F: Matrix-assisted laser desorption /ionization time-of-flight mass spectrometry of wide mass band (WB) peptide mixture deposited into crushed aCCa crystals corresponding to Figure $2 \mathrm{~B}$ as a function of laser power for the protonated species, avera.

The laser power for the protonated peaks, in general, was higher than the sodiated peaks and also were lower in general for acidic peptides relative to the entire spectra of peptides in the WB mix. The protonated/sodiated peaks for aCCa only or aCCa I (open circle, open square) analytes in protonated or sodiated form were also greater, with the population being split evenly between the two forms. With iodine alone, the protonated peak dominated relative to the sodiated peak (blue line versus blue line in graph $3 F$ ).

\section{Conclusion}

We have demonstrated that ion intensities could be enhanced by the synthesis of long-chain matric species (computational based on gas-phase proton affinities) or deposition of alpha hydroxycinnamic acid onto structured surfaces or deposition upon crystallized and crushed aCCa as a dual deposition system. Alternatively, by the addition of an ultra-low dose of molecular iodine, the ion intensities were always greater than under matrix-assisted laser desorption/ ionization-time of flight conditions, which is attributed to enhanced gas-phase desorption. As the radical species were not detected, only protonated or sodiated (or to a lesser degree potassiated) pseudo molecular ions, it appears the iodine promoted the desorption step to a greater degree than ionization, but also significant enhancement of the ionization step. Using the pseudo molecular ion peak intensities of the protonated and sodiated species for a wideband peptide mixture and comparison to aCCa only, it is suggested that $2 / 3$ of the enhancement is due to desorption and $1 / 3$ due to enhancement in the ionization step, where the molecular 
iodine is acting as a photosensitizer by transferring charge to proton and sodium ions rather than forming iodine or matrix adducts. Further analysis of the dependence of laser power suggests that ion transfer occurs between 5-7 eV. The data collectively indicate that iodine desorption involves bulk exciton decay at the surface and thermally assisted $\mathrm{H}^{+}$ diffusion that is trapped at the surface due to surface defects resulting in metastable decay. This would also account for the observation that aCCa deposited onto crushed aCCa crystals resulted in greater ion intensities.

\section{Acknowledgment}

The authors are grateful to the National Science Foundation (NSF) Centers of Research Excellence in Science and Technology (NSF CREST, HRD-0734850, and CBET MRI-0821370) at the Texas A\&M University-Kingsville. The Robert C Welch (AC-0006) Departmental Grant is also duly acknowledged.

\section{Author Contribution}

S. Bashir conducted the research, wrote the first draft. S. R. Chava validated all the structures and J. Liu drew Scheme 1 and 2, assisted with the hypothetical synthesis scheme and statistical analysis on other figures 2B-4.

\section{Tribute}

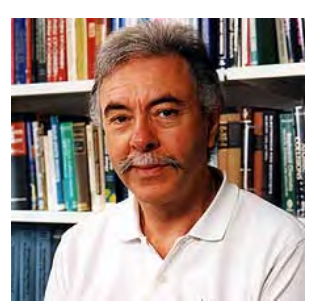

† It is with profound sadness with which SB writes this acknowledgment. There is a word in the Old Testament, salah, meaning to pause, to reflect, and to think. I deeply miss Peter, but I will never forget the important lessons he taught me about being a scientist, teacher, and human being. JL Liu writes I never met Dr. Derrick, but his knowledge to advance science and responsibility to educate the young generation has been an inspiration in my academic career. We shall never forget the sweet, courageous, and dedicated man, whose name will always linger in our thoughts. Thank you, Dr. Peter Derrick, and God Speed. On February 15, 2020, I also lost my father due to SARS2, due to the lockdown I was unable to travel or attend the funeral or meet with my mother or brother and sisters, and six months later at the time of writing this tribute the situation has not changed. This article is in memory of all of us who have lost a loved one due to SAR2.

\section{References}

1. Knochenmuss (2002) A quantitative model of ultraviolet matrix-assisted laser desorption/ionization. J Mass Spectrom 37: 867-877.

2. Zenobi, Richard Knochenmuss (1998) Ion formation in MALDI mass spectrometry. Mass Spec Rev 17(5): 337366.

3. Najam, Rainer M, Heigl N, Szabo Z, Vallant R, et al. (2008) Nano-structured support materials, their characterisation and serum protein profiling through MALDI/TOF-MS. Amino Acids 34: 279-286.

4. Patrick D Setz, Richard Knochenmuss (2005) Exciton mobility and trapping in a MALDI matrix. J Phys Chem A 109(18): 4030-4037.

5. Anthony Halfacre J, Kyle A Beran, Nicholas R Beller, Mario Gomez, Brandon L Rebber, et al. (2007) Theoretical Investigation of the Proton Affinity and Gas-Phase Basicity of Neutral x,y-Dihydroxybenzoic Acid and its Derivatives Eur J Mass Spectrom 12: 385-396.

6. Sérgio Filipe Sousa, Pedro Alexandrino Fernandes, Maria João Ramos (2007) General performance of density functionals. J Phys Chem A 111(42): 10439-10452.

7. Zhi Y, Xiao-Chong Yu, Qihuang Gong, Lan Yang, Yun-Feng Xiao (2005) Single Nanoparticle Detection Using Optical Microcavities Adv Mater 29(12): 1492-1496.

8. Fassett (1990) Manufacturers' Literature. Anal Chem 62(6): 386-389.

9. Price DM, Sajid Bashir, Peter R Derrick (1999) Sublimation properties of $\mathrm{x}, \mathrm{y}$-dihydroxybenzoic acid isomers as model matrix assisted laser desorption ionisation (MALDI) matrices. Thermochim Acta 327(12): 167-171. 